\title{
Multilayer Artificial Approach for Estimating Optimal Solar PV System Power Using the MPPT Technique
}

\author{
Aouatif IBNELOUAD ${ }^{1 *}$, Abdeljalil EL KARI ${ }^{1}$, Hassan AYAD ${ }^{1}$, Mostafa MJAHED ${ }^{2}$ \\ ${ }^{1}$ Laboratory of Electrical Systems, Energy Efficiency and Telecommunications, Department of \\ Applied Physics, Cadi Ayyad University, Faculty of Sciences and Technologies, Marrakech, Morocco \\ aouatif.ibnelouad@ced.uca.ma (*Correspondingauthor), a.elkari@uca.ma,h.ayad@uca.ma \\ ${ }^{2}$ Department of Mathematics and Systems, Royal School of Aeronautics, Marrakech, Morocco \\ mjahed.mostafa@gmail.com
}

\begin{abstract}
Artificial neural networks (ANNs) are widely recognized as technologies for solving complex problems and they have been applied effectively in several fields. Today, ANNs go beyond the limits of the conventional approaches by extracting the desired information directly from the available data. The prediction and estimation performance for this technique in the context of PV systems is essential for choosing it because it has a significant impact on the quality of the production of electrical energy and therefore on the efficiency of the PV system. After analyzing the DC/DC control loop which includes the maximum power point (MPP) controller, this work focuses on the analysis of the estimation and the prediction of errors through the new "Multi-Input Multi-Layer One-Output (MIMLOO)" approach using the ANN technique. It was developed in a model based on either a single hidden layer or two hidden layers by using a scheme of multiple inputs to one output (a "many-to-one" relationship). Its goal is to estimate the performance of the employed method by modifying the number of hidden layers and the types of algorithms that were implemented in the context of the analysed PV system. For this purpose, the performance of the proposed approach was estimated based on a real database acquired from the "SOLON 55W" PV panel. The simulation results obtained in MATLAB / Simulink show the efficiency and robustness of this approach for the "SOLON 55W" PV system.
\end{abstract}

Keywords: Artificial neural networks (ANNs), Multi-Input Multi-Layer One-Output (MIMLOO), Maximum power point controller (MPP), PV system, DC/DC converter.

\section{Introduction}

Currently the consumption of energy is increasing due to the trend of rapid industrialization and demographic change, which leads to the consumption of the stock of energy coming from fossil fuels (petroleum, natural gas etc.), which leads to the research and development of new sources of renewable energy. Solar energy is the most important source because photovoltaic converters directly convert energy from solar radiation into electrical energy (Ouada et al., 2012). Photovoltaic solar energy comes from the direct transformation of a part of solar radiation into electrical energy. This energy conversion takes place through a so-called photovoltaic (PV) cell based on a physical phenomenon called the electromotive force which occurs due to the difference in electric charge concentration. Solar radiation leads to the generation of free charge. The voltage generated may vary depending on the material used for the manufacturing of the cell. The association of several PV cells in series / parallel gives rise to a photovoltaic generator (GPV) which has a non-linear currentvoltage (I-V) characteristic exhibiting a maximum power point (Hananou \& Rouabah, 2014). The maximum power point parameter is indispensable as it has been recognized as a crucial parameter in the design of solar energy extracting the PV power, and in the prediction of optimal power extracted. Unfortunately, the PV generator is a non-linear system and depends on the meteorological conditions (solar radiation, temperature) which prevented the extraction of generalized maximum power of solar energy in an efficient manner. Conventional energy is increasingly in demand because of the rapid development of the social economy and the growing energy needs. Nowadays, the world is facing environmental pollution and ecological deterioration because there is heavy use of conventional energy sources.

The development and application of new energies is becoming the inevitable trend of world development. Solar energy as green energy has become one of the most promising and important new energy sources among all renewable energies because it does not create pollution, noise and for other unique benefits. The non-linearity of the solar cell, the maximum voltage of the power point will change with the change of light intensity and ambient temperature. In order to improve the conversion efficiency for solar cells, it is necessary to follow the output of the maximum power (Han et al., 2016). In most photovoltaic energy production systems, there is a technique or algorithm called "Maximum Power Point Tracking" (MPPT), which results in maximum 
power point tracking. This technique, as its name suggests, tracks the MPP over time and allows one to get the maximum power that the panel can provide. The MPPT aims to improve and optimize the operation of photovoltaic systems. It allows one to make a gain that can reach $25 \%$, hence its importance. Technically, MPPT uses an interface between the panel and the load which is usually a power conversion device (Boukli, 2011; Soria \& Makhlouf, 2017).

Artificial neural network (ANN) is a collection which includes an enormous number of interrelated processing elements, known as neurons, employed together to solve a problem. Neural networks have a notable capability of handling complex or inexact data, which is too multifaceted to be solved by other advanced techniques. It is widely used for modelling complex problems and well suited for forecasting. ANNs are widely applied due to some basic properties of this technique: (1) The ability to process the problems for which the violation of any constraints by some of the neurons would not affect the overall output; (2) The capability to extrapolate from historical facts and generate predictions and (3). The capability of the ANNs to solve complex and non-linear problems (Murat, Y.S., 2006). Over the past two decades, the Artificial Neural Network (ANN) has been the most common artificial intelligence (AI) technique used. It can model nonlinear and complex systems thanks to its ability to regulate weights and biases. There are many ANN structures, such as the multilayer perceptron (MLP) (Alsina et al., 2016), the recurrent neural network (RNN) (Hajdarevic et al., 2015) and radial basis function (RBF) network (Wu et al., 2012). These various structures based on the flexible artificial neural network and very versatile with regard to their applications. The application of the ANN model to estimate the optimum power of the PV generator, using various meteorological variables, is frequently discussed. Weather variables are the key parameters that are used for measuring the maximum power for a selected PV generator.

Regarding the rapid development of solar energy in the economy of cities, few cities have the measuring equipment used for the precise measurement of various parameters. In addition, the data related to the extraction of PV power to switch to a non-polluting environment, as such predictive approaches become interesting (Timilsina, Kurdgelashvili \& Narbel, 2012) The power can be estimated by applying models based on artificial intelligence (Gani et al., 2016; Li, Wang \& Wei, 2018). A frequently discussed problem concerning correlation models predicting the optimum power of the SOLON 55W PV panel is the optimum choice of the nature and number of the input parameters (irradiation and temperature). Almost all the models developed in the contributions to the scientific literature are based on a unique structure where they are trained to estimate the optimum power values using dependent or independent climate variables, collected in recent years, without specifying the validity subspaces, and thereafter without modelling each subspace with an appropriate sub-model. Using the single-structure model can decrease the accuracy of the estimate and slow down the learning phase, resulting in divergence and instability of the predictive model. The solution therefore consists in dividing the exploitation space using the multi-model approach.

This article follows the methodology presented by several contributions for the literature (Manan et al., 2018; Kumar et al., 2016) highlighting their efficiency for the SOLON 55w PV panel. The key elements of the innovation brought about by this article concern the development of a new hybrid machine learning algorithm based on the multimodel approach and the artificial neural network to accurately estimate the optimal power of the SOLON 55W photovoltaic panel. The choice to use the multi-model approach is due, on the one hand, to its ability to model nonlinear dynamic systems such as the case of optimal power, and, on the other hand, to its popularity with regard to the simplicity of the representation of the nonlinear system obtained by the decomposition of the space of operations into several subspaces, allowing the description of the system by several sub-models with a simple structure. In addition, the artificial multi-neural approach is trained to estimate the optimal power using the most relevant inputs determined by a sensitivity analysis. Using this new approach speeds up the training process and ensures the reliability of the model throughout the year. In addition, it is a good tool for monitoring the quality of the estimate and detecting if it decreases. Subsequently, it is easy to locate the sub-model where there is a deficiency and try to adjust its parameters without affecting the parameters of the other sub-models. This is not possible when single-structure models are used.

https://www.sic.ici.ro 
This paper is organized as follows. Section 2 describes the proposed model of the SOLON $55 \mathrm{~W}$ PV panel. Section 3 sets forth the adopted approach using ANN and SUB-Model. Further on, Data Pre-processing is outlined in Section 4. Section 5 presents the simulation results and Section 6 includes the conclusions of this paper.

\section{PV Module Modelling}

The PV generator is a set of photovoltaic panels connected in series and in parallel. Each panel is made up of photovoltaic cells that convert photons into voltage and current. $I_{p h}$ in parallel with a diode with a series resistance $\left(R_{s}\right)$ and a parallel resistance $\left(R_{p}\right)$ are illustrated in Figure 1, which represents the mathematical model of a cell. (Ndoye et al., 2009) .

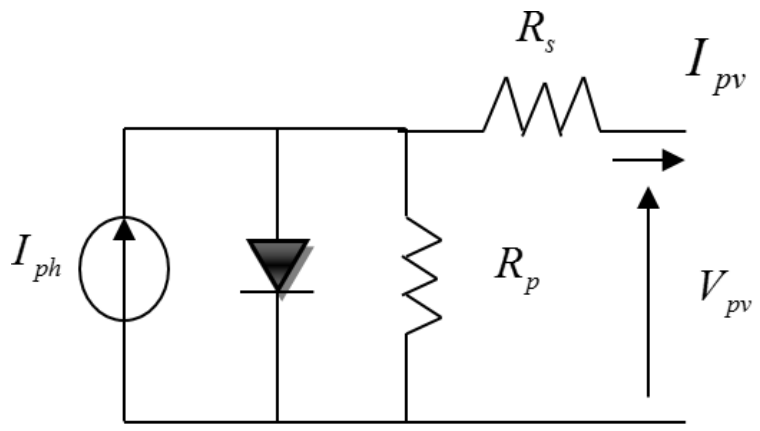

Figure 1. Mathematical model of a solar cell

Under darkness, the diode equation is given by (Soteris, 2009; Luque \& Hegedus, 2003):

$$
I_{d}=I_{s}\left(e^{\left(q V_{p v} / n k T\right)}-1\right)
$$

Under illumination, one obtains:

$$
I_{p v}=I_{p h}-I_{s}\left(e^{\frac{q V_{p v}}{n k T}}-1\right)
$$

So, the final equation becomes:

$$
I_{p v}=I_{p h}-I_{s}\left(e^{\frac{q V_{p v}}{n k T}}-1\right)-I_{p}
$$

With: $I_{p}=\frac{V_{p v}+R_{s} I_{p v}}{R_{p}}$

where: $I_{p}$ is parallel resistance current, $I_{p h}$ is current Generator, $I$ is reverse saturation current of the diode, $\mathrm{q}$ is charge of the electron $\left(1.610^{-19} \mathrm{C}\right), k$ is Boltzmann's constant $\left(1.810^{-23} \mathrm{~J} / \mathrm{k}\right), \mathrm{n}$ is the nonideality factor of the solar cell $(1<\mathrm{n}<2), T$ is the temperature of the junction in $k, V_{p v}$ is the output voltage, and $I_{p v}$ represents the output currents.

\subsection{Characteristics of the SOLON 55W PV Panel}

Figure 2 shows the SOLON 55W monocrystalline PV panel. It is made up of 36 cells connected in series and in parallel, with bypass and non-return diodes. The respective characteristics, namely $\mathrm{I}=$ $f(V)$ and $P=f(V)$ of this panel for the real case are given in Figure 3 and Figure 4.

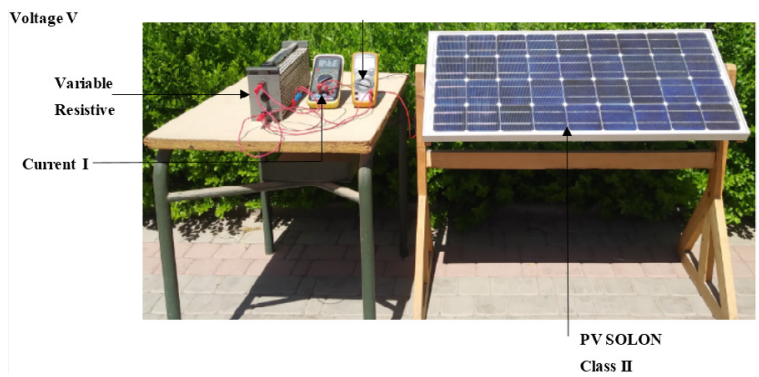

Figure 2. SOLON 55W monocrystalline PV panel

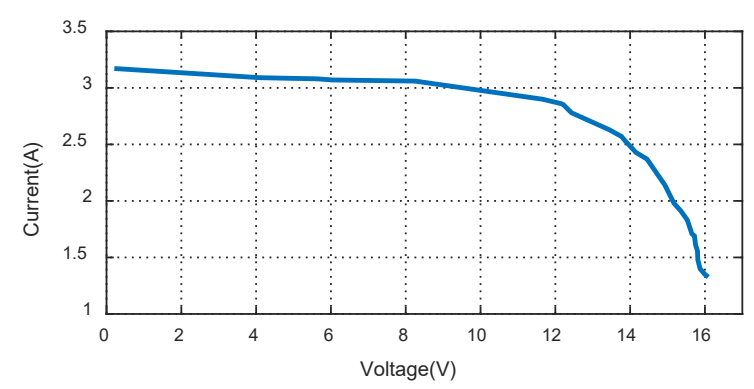

Figure 3. Characteristic $I=f(V)$ of the Solon $55 \mathrm{~W}$ PV panel

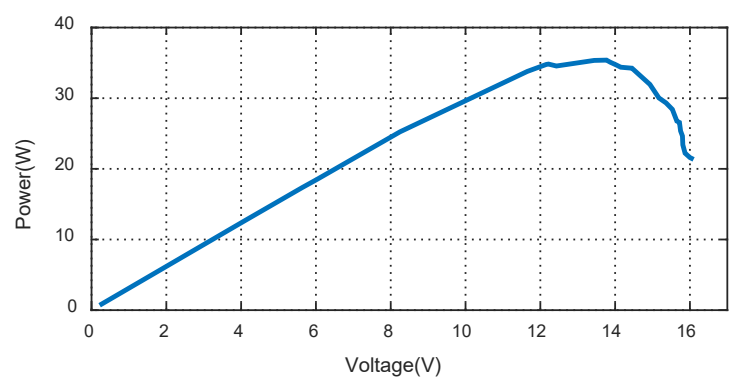

Figure 4. Characteristic $\mathrm{P}=\mathrm{f}(\mathrm{V})$ of the Solon $55 \mathrm{~W}$ PV panel

\subsection{Simulation and Validation of the "SOLON 55W" Photovoltaic Model}

The mathematical model of the photovoltaic generator is always represented by equations using the output and the inputs, there is a current source containing the lighting value connects in parallel with a diode and in parallel with a resistor in series with another resistor (Takun, Kaitwanidvilai \& Jettanasen, 2011). The SOLON 55W type PV module in Figure 2. 
The parameter values of the SOLON 55W PV panel used in the simulation are shown in Table 1. The simulation of the PV module and of the subsystem of the considered PV module considered for different temperature and irradiation values is illustrated in Figure 5 and Figure 6, respectively. The PV module has non-linear characteristics as it is shown in Figure 7 and Figure 8.

Table 1. Manufacturer's data sheet for the SOLON 55W Class II Model

\begin{tabular}{|l|l|}
\hline \multicolumn{1}{|c|}{ Technical Specification } & \multicolumn{1}{c|}{ Data Sheet } \\
\hline Type & SOLON 55W \\
\hline Safety Class & II \\
\hline Maximum System Voltage & $600 \mathrm{~V}$ \\
\hline Rated power $\mathrm{P}_{\max }$ & $55 \mathrm{~W}$ \\
\hline Rated Current $\mathrm{I}_{\operatorname{mpp}}$ & $3.15 \mathrm{~A}$ \\
\hline Rated Voltage $\mathrm{V}_{\text {mpp }}$ & $17.5 \mathrm{~V}$ \\
\hline Short Circuit Current $\mathrm{I}_{\mathrm{sc}}$ & $3.41 \mathrm{~A}$ \\
\hline Open Circuit Voltage $\mathrm{V}_{\mathrm{oc}}$ & $21.8 \mathrm{~V}$ \\
\hline
\end{tabular}

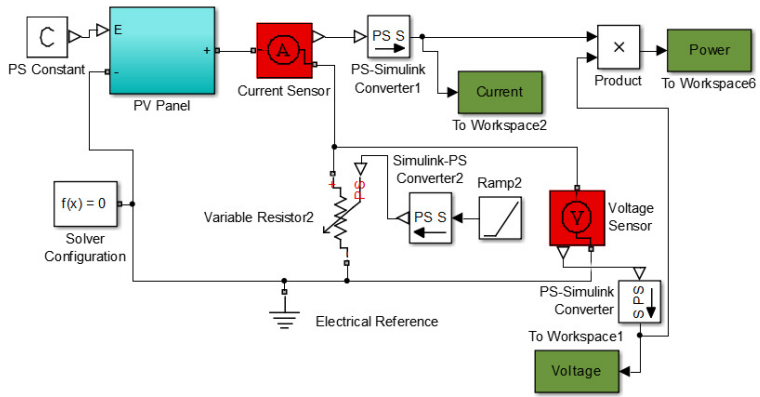

Figure 5. PV module under MATLAB / Simulink

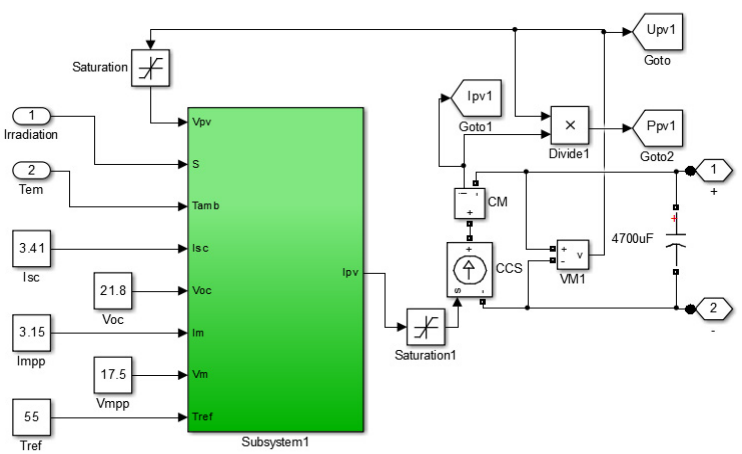

Figure 6. The subsystem of the considered PV module under MATLAB / Simulink

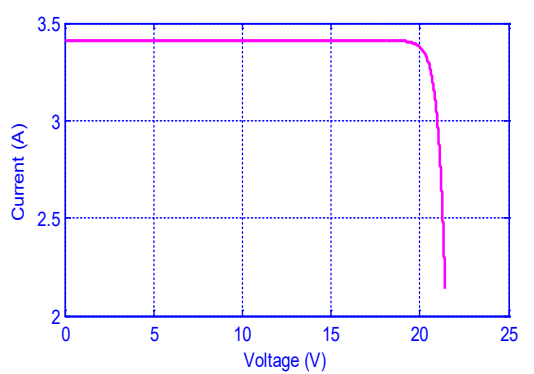

Figure 7. PV module Characteristic $I=f(V)$ under MATLAB / Simulink

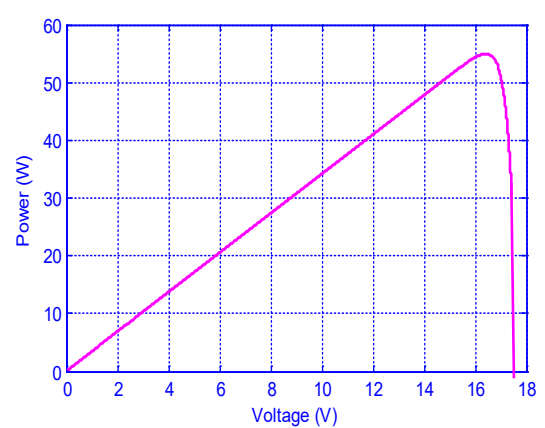

Figure 8. $P V$ module Characteristic $P=f(V)$ under MATLAB / Simulink

\section{Artificial Neural Networks (ANN)}

In the early 1940s, (McCulloh \& Pitts, 1943) perceived the neural network and became popular around 1985, when Rumelhart et al. (1986) introduced the method of "Back-Propagation" to train the network (Pushpi \& Dilip, 2018). Subsequently, it was applied in several areas of research and a lot of work was done. ANNs have been used successfully to solve complex problems in various fields of application, including identification, classification and control systems, particularly in the field of solar energy estimation (Sözen et al., 2004; Kok et al., 2012). Nowadays, to solve the difficult problems of conventional computers or human beings, ANNs can be trained. By contrast, ANNs overcome the limitations of the conventional approach by extracting the information sought directly from the data. An ANN functions as a "black box" model (Kalogirou, 2004), (Sözen et al., 2004). "Back Propagation (BP)" has been widely adopted as a successful learning rule for finding appropriate values of weights for ANNs. The MLP consists of various layers: one input layer, one hidden layer or several hidden layers whose exits are not visible and exits. These layers are based on interconnected neurons using feed-forward weighted links (Taright \& Hubin, 1998). All these neurons perform the same operation, the sum of their weighted inputs (equation 4). Then, the result is applied to a nonlinear function called the activation function and generally based on the sigmoid function (equation 5) (Mellit et al., 2008).

$$
\begin{aligned}
& y_{j}=f\left[\left(\sum_{i} w_{i j} x_{i j}\right)-b_{j}\right] \\
& f(x)=\frac{1}{\left(1+e^{-x}\right)}
\end{aligned}
$$


Where $y_{j}$ is the output of the processing block; $x_{i j}$ are the inputs; $\mathrm{w}_{i j}$ is the synaptic weight coefficient of the $i^{\text {th }}$ input of the processing block, and $b_{j}$ is the bias.

\subsection{Description of the Multi-Model}

The idea of multi-model approach is based on the decomposition of a complex system into subsystems (Madani \& Thiaw, 2007; Jalal et al., 2019). Each subsystem is based on predetermined data and then gives individual solutions that help the overall system. This approach represents the projection of the proposed photovoltaic PV system under two models of the same MPPT control using ANN. Each model is made up of sub-models with different hidden layers. The main goal is to split these two models in order to obtain the best model, as the output is concerned, and also with regard to precision and the minimization of errors between the two models. The architecture of the multimodel technique is shown in Figure 9 below:

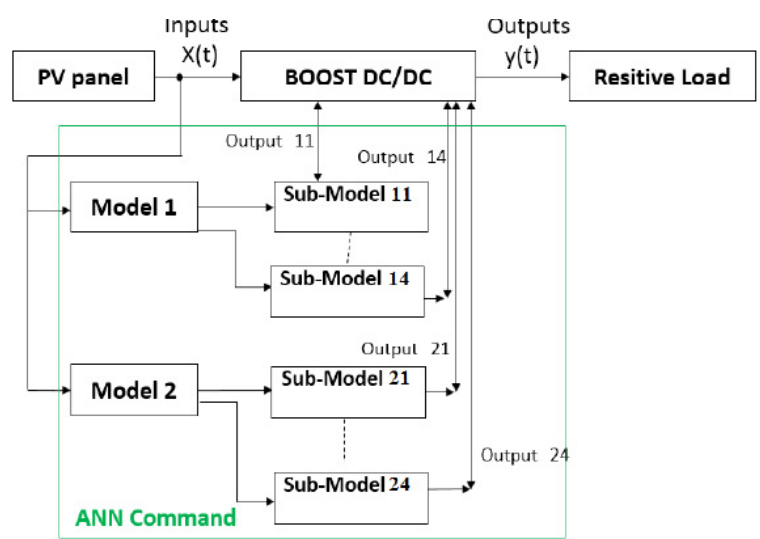

Figure 9. Architecture of the multi-model technique

For each model, there are four sub-models which give 4 outputs, the aim is to find the best solution. $F(x(t))$ represents the summation of the different sub-models of model $1 . \mathrm{G}(\mathrm{x}(\mathrm{t}))$ represents the summation of the different sub-models of model 2. To determine the best solution, the errors for each sub-model are calculated. The system output is subtracted for each model. After that, the submodel that gives a minimum error solution is the best sub-model in the relevant model.

$$
\begin{aligned}
& F\left((x(t))=\sum_{k=1}^{n} \text { Output }_{1 k}(t)=\sum_{k=1}^{n} f_{1 k}(x(t))\right. \\
& G\left((x(t))=\sum_{k=1}^{n} \text { Output }_{2 k}(t)=\sum_{k=1}^{n} f_{2 k}(x(t))\right. \\
& \text { Error }_{1}=\varepsilon_{1}(t)=y(t)-F(x(t)) \\
& \text { Error }_{2}=\varepsilon_{2}(t)=y(t)-G(x(t))
\end{aligned}
$$

\subsection{Description of the Artificial Multi- layered Approach}

This part presents the architecture of a new machine learning algorithm called "Artificial Multi-Layer Approach". This intelligent approach is based on the association between ANN and multi-model technique for the improvement of an intelligent hybrid model which includes the characteristics of the two techniques described in the previous sections. This technique is created to increase the accuracy of hourly estimates for optimum power over Figure months of the year by outputting this command to the DC / DC boost converter in order to extract maximum power with minimum error between the output power of the converter and the power of the PV panel, thus overcoming the problems linked to the absence of hourly measurements for the different characteristics of the PV panel. On the other hand, these estimates make it possible to choose the best algorithm used on the ANN command with the number of hidden layers used as well as the number of neurons taken. Figure 10 shows the Multi-Input Multi-Layer One-Output (MIMLOO) model architecture which is based on this new approach to estimate the optimal power using two inputs (Temperature and Solar irradiation).

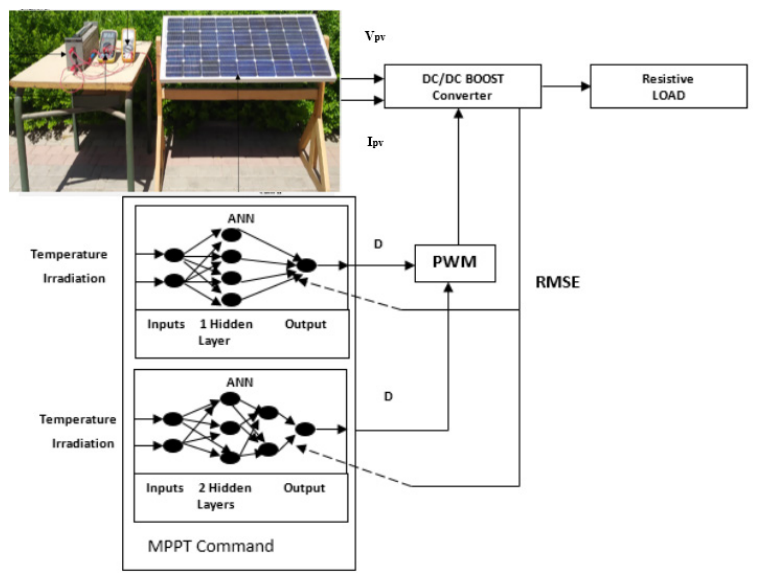

Figure 10. Multi-Input Multi-Layer One-Output (MIMLOO) architecture

For this, two models were created, each of them consisting of four sub-models. Each sub-model is associated with the same database (irradiation and temperature) measured for meteorological data for four months (months 5, 6, 7 and 9) of the year 2019. The first model is based on a single hidden layer with 4 sub-models. Each sub-model is made up of a different number of neurons (4, 6, 7 and 9). The second model is based on two hidden layers with 4 sub-models. Each sub-model is made up 
of a different number of neurons for each hidden layer $(\{2,1\} ;\{2,3\} ;\{3,2\} ;\{5,4\})$.

The training of each sub model $\mathrm{k}$ was performed using half of the database of its associated month $\mathrm{k}$, and the other half was used to test its efficiency in order to estimate the time series.

After the distribution of the database, one starts the creation and configuration of these sub-models by following the different steps described in Figure 11.
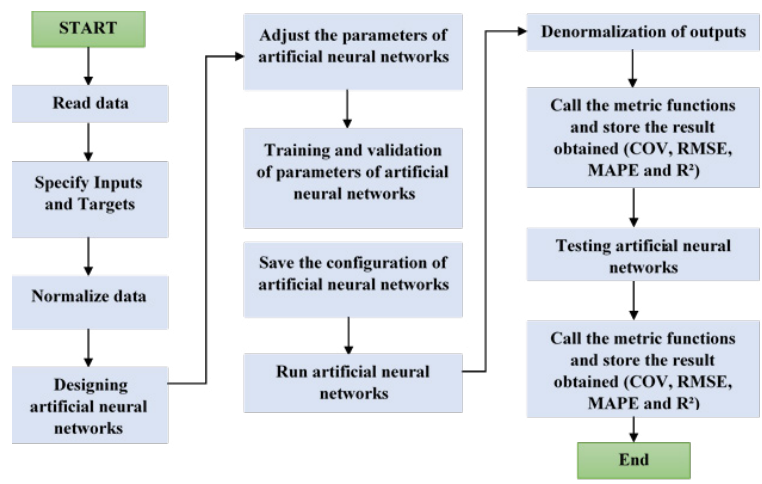

Figure 11. Flowchart of supervised artificial neural networks model (Jalal et al., 2019)

\section{Data Pre-processing}

In this study, a 55W SOLON class II PV panel was used, which is illustrated in Section 2. This panel was connected in series with an Amperemeter to measure current, and in parallel with a voltmeter to measure voltage. A DC/DC BOOST converter was employed to adapt the operating point of the $\mathrm{PV}$ with its maximum power point connected with a resistive load. To measure the radiation, a Pyranometer of the type "KIPP \& ZONEN" was used.

The collection of this data spans for four months. Each day of these four months, the change in temperature and solar radiation was registered for 10 hours a day. The data acquisition for the different variables for each week (solar irradiation, temperature, voltage and current) is represented successively in Figure 12:

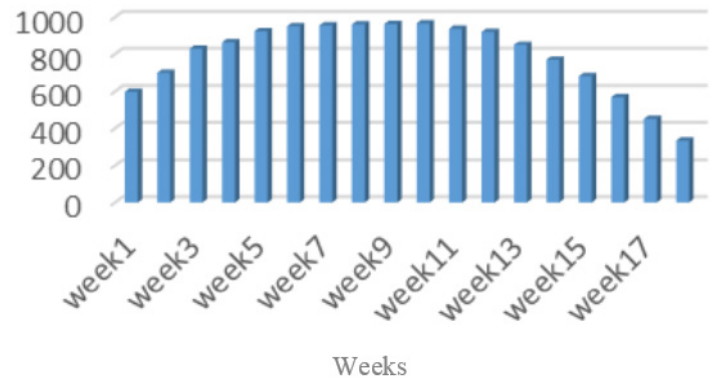

(a)

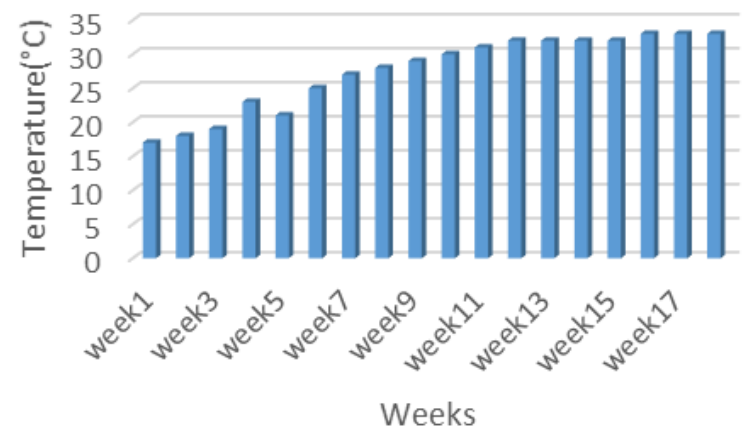

(b)

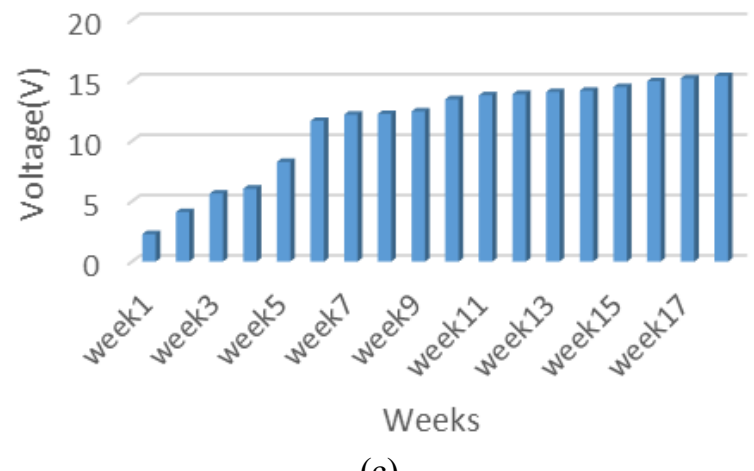

(c)

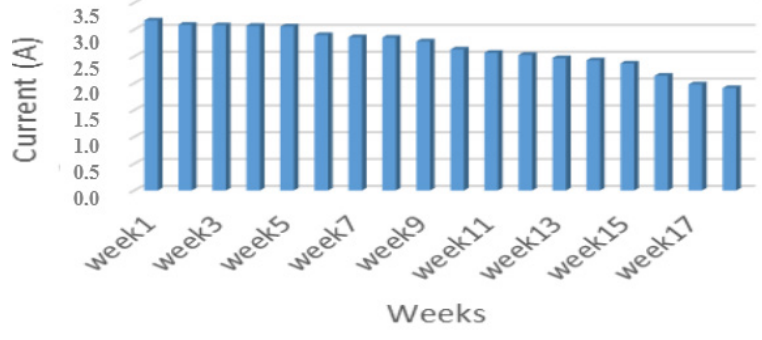

(d)

Figure 12. Acquiring panel data: (a) Solar irradiation " $\left(\mathrm{w} / \mathrm{m}^{2}\right)$ ", (b) Temperature " $\left({ }^{\circ} \mathrm{C}\right)$ ", (c) Voltage " $(\mathrm{V})$ ", (d) Current "(A)"

\subsection{Performance Metrics}

Root Mean Squared Error (RMSE): the root mean squared error (RMSE) is a frequently used measure for the differences between values (sample or population values) predicted by a model or estimator and observed values (Hyndman \& Koehler, 2006). The RMSE is always nonnegative, and a value of 0 (almost never reached in practice) would indicate a perfect fit to the data. In general, a lower root mean square is better than a higher one. However, comparisons between different types of data would not be valid because the measure depends on the scale of the numbers used. RMSE is the square root of the mean of the squared errors. The effect of each error on RMS is proportional to the size of the squared error; thus, larger errors have a disproportionate effect on the RMSE. Therefore, RMSE is sensitive to outliers (Pontius et al., 2008; Willmott \& Matsuura, 2006).

https://www.sic.ici.ro 


$$
R M S E=\sqrt{\left(\frac{1}{p}\right) \sum_{j}\left(t_{j}-o_{j}\right)^{2}}
$$

Absolute Fraction of Variance $\left(\mathrm{R}^{2}\right)$ : The coefficient of determination (R-squared) indicates the proportional amount of change in the response variable y explained by the independent variables $\mathrm{x}$ in the linear regression model. The higher the R-squared, the more variability is explained by the linear regression model. $\mathrm{R}^{2}$ expresses the proportional relationship between the actual outputs and the outputs of the ANNs (the higher the $\mathrm{R}^{2}$, the more variability is explained by the linear regression model).

$R^{2}=1-\left(\frac{\sum_{j}\left(t_{j}-o_{j}\right)^{2}}{\sum_{j}\left(o_{j}\right)^{2}}\right)$

Coefficient de variation (COV): The coefficient of variation is a measure of relative dispersion. RMS is defined as the absolute value of the coefficient of variation and is often expressed as a percentage.

$$
C O V=\frac{R M S E}{o_{\text {mean }}}
$$

Average error in absolute percentage (MAPE): is a network performance function. It measures network performance as the average of the absolute errors.

$$
M A P E=\frac{o-t}{o} * 100
$$

where $t$ is the target value, $o$ is the output value and $\mathrm{p}$ indicates the number of patterns.

\section{Results and Discussion}

Table 2 shows the results of simulations for

\begin{tabular}{|c|c|c|c|c|c|c|c|c|c|c|c|}
\hline \multirow[b]{2}{*}{ Algorithm } & \multirow[b]{2}{*}{$\begin{array}{l}\text { Sub- } \\
\text { Model }\end{array}$} & \multirow[b]{2}{*}{$\begin{array}{c}\text { Hidden } \\
\text { layers }\end{array}$} & \multirow{2}{*}{$\begin{array}{c}\text { Number of } \\
\text { Neurons } \\
\{1 \text { hidden } \\
\text { layer, } 2 \\
\text { hidden } \\
\text { layers\} }\end{array}$} & \multicolumn{4}{|c|}{ Training } & \multicolumn{4}{|c|}{ Testing } \\
\hline & & & & $\begin{array}{l}R^{2} \\
(\%)\end{array}$ & $\begin{array}{c}\text { RMSE } \\
(\%)\end{array}$ & $\begin{array}{c}\text { MAPE } \\
(\%)\end{array}$ & $\mathrm{COV}$ & $\begin{array}{l}R^{2} \\
(\%)\end{array}$ & $\begin{array}{c}\text { RMSE } \\
(\%)\end{array}$ & $\begin{array}{c}\text { MAPE } \\
(\%)\end{array}$ & $\mathrm{COV}$ \\
\hline \multirow{8}{*}{ SCG } & 1 & 1 & 4 & 94.908 & 21.614 & 12.742 & 0.1711 & 98.786 & 9.635 & 5.899 & 0.0763 \\
\hline & 2 & 1 & 6 & 94.593 & 20.178 & 12.139 & 0.1598 & 97.571 & 23.716 & 8.144 & 0.1878 \\
\hline & 3 & 1 & 7 & 96.61 & 20.351 & 12.271 & 0.1611 & 80.426 & 13.183 & 7.756 & 0.1044 \\
\hline & 4 & 1 & 9 & 92.012 & 19.734 & 12.104 & 0.1563 & 99.936 & 11.797 & 2.813 & 0.0934 \\
\hline & 5 & 2 & $\{2,1\}$ & 88.957 & 20.152 & 10.343 & 0.1596 & 96.527 & 14.656 & 7.372 & 0.1160 \\
\hline & 6 & 2 & $\{2,3\}$ & 88.447 & 46.660 & 23.391 & 0.3695 & 99.159 & 20.092 & 5.278 & 0.1591 \\
\hline & 7 & 2 & $\{3,2\}$ & 88.704 & 23.627 & 6.285 & 0.1871 & 97.738 & 18.476 & 9.970 & 0.1463 \\
\hline & 8 & 2 & $\{5,4\}$ & 98.913 & 56.92 & 2.368 & 0.0451 & 97.637 & 9.410 & 5.553 & 0.0745 \\
\hline \multirow{8}{*}{ LM } & $\begin{array}{c}\text { Sub- } \\
\text { Model } 1 \\
\end{array}$ & 1 & 4 & 99.86 & 12.115 & 0.775 & 0.0959 & 95.49 & 11.219 & 2.727 & 0.0888 \\
\hline & 2 & 1 & 6 & 93.97 & 22.597 & 1.708 & 0.1789 & 90.38 & 18.089 & 2.364 & 0.1432 \\
\hline & 3 & 1 & 7 & 98.34 & 10.156 & 4.949 & 0.0804 & 98.60 & 14.294 & 1.310 & 0.1132 \\
\hline & 4 & 1 & 9 & 93.32 & 14.993 & 15.761 & 0.1187 & 99.94 & 10.921 & 0.937 & 0.0865 \\
\hline & 5 & 2 & $\{2,1\}$ & 87.55 & 20.922 & 12.320 & 0.1657 & 83.51 & 8.048 & 2.794 & 0.0637 \\
\hline & 6 & 2 & $\{2,3\}$ & 95.75 & 12.937 & 6.626 & 0.1024 & 89.06 & 9.991 & 3.363 & 0.0791 \\
\hline & 7 & 2 & $\{3,2\}$ & 94.53 & 30.704 & 1.340 & 0.2431 & 99.01 & 15.393 & 5.944 & 0.1219 \\
\hline & 8 & 2 & $\{5,4\}$ & 95.33 & 26.851 & 3.841 & 0.2126 & 94.05 & 22.138 & 12.297 & 0.1753 \\
\hline \multirow{8}{*}{ CGP } & $\begin{array}{c}\text { Sub- } \\
\text { Model } 1\end{array}$ & 1 & 4 & 87.51 & 19.879 & 10.844 & 0.1574 & 93.47 & 14.195 & 6.950 & 0.1124 \\
\hline & 2 & 1 & 6 & 39.34 & 48.866 & 25.192 & 0.3869 & 78.575 & 10.125 & 3.911 & 0.0802 \\
\hline & 3 & 1 & 7 & 35.52 & 10.3847 & 14.383 & 0.8223 & 95.216 & 10.011 & 1.242 & 0.0793 \\
\hline & 4 & 1 & 9 & 91.06 & 25.428 & 7.178 & 0.2013 & 74.947 & 10.289 & 2.111 & 0.0815 \\
\hline & 5 & 2 & $\{2,1\}$ & 50.24 & 41.801 & 19.723 & 0.3310 & 97.007 & 18.241 & 10.475 & 0.1444 \\
\hline & 6 & 2 & $\{2,3\}$ & 62.32 & 38.965 & 19.945 & 0.3085 & 66.241 & 13.261 & 13.76 & 0.1050 \\
\hline & 7 & 2 & $\{3,2\}$ & 45.11 & 53.706 & 25.994 & 0.4253 & 47.017 & 14.166 & 15.64 & 0.1122 \\
\hline & 8 & 2 & $\{5,4\}$ & 97.86 & 18.135 & 1.767 & 0.1436 & 80.358 & 11.019 & 5.441 & 0.0873 \\
\hline
\end{tabular}
the different values of the coefficients already

Table 2. ANN Training and Testing Stage Error Values 
calculated using the MATLAB / Simulink software for the SOLON 55W panel. Figure 13 and Figure 14 illustrate the simulation of the different variables of the proposed model from Table 2 .

After determining all the structures of the submodels, three Back-propagation (BP) algorithms are applied to adjust the weights and biases of the developed model in order to achieve best optimization algorithm that can be used to train the final neural sub-models. Figures 13 and 14 below illustrate the evolutions of the statistical indicators COV, RMSE and MAPE, and of the correlation coefficient $\mathrm{R}^{2}$ in the two phases of each submodel and each learning algorithm. Based on the values obtained in the two phases of all the submodels with different statistical indicators, it can be noticed that the Levenberg-Marquardt (LM) algorithm is the most suitable one for training all the sub-models from among the other learning algorithms. "Pola-Ribiere Conjugate Gradient (CGP)", and Scaled Conjugate Gradient (SCG)" learning algorithms give large values in terms of statistical criteria used (COV, RMSE, MAPE) and less precision in terms of correlation coefficient $\left(\mathrm{R}^{2}\right)$ compared to the $\mathrm{LM}$ algorithm, except for certain sub-models like those illustrated in Figures 13 and 14, where they give results closer to those obtained by the LM algorithm.

The results obtained allow the generalization of the LM learning algorithm for adjusting the weights and biases of all the sub-models developed. These results demonstrate that the LM algorithm is the best algorithm to use in the ANN method. The figures below represent the means for each coefficient. Each figure demonstrates the speed and efficiency of the LM algorithm in comparison with other algorithms. $\mathrm{R}_{\text {mean }}^{2}=0.84$ for $\mathrm{LM}, \mathrm{R}_{\text {mean }}^{2}=0.76$ for SCG and for CGP $\mathrm{R}_{\text {mean }}=0.40$. So, for the LM algorithm, this coefficient is closest to 1 . As the RMSE coefficient is concerned for the LM algorithm, $\mathrm{RMSE}_{\text {mean }}=1.89$, for the SGC algorithm, $\mathrm{RMSE}_{\text {mean }}=2.22$, and for the CGP algorithm, $\mathrm{RMSE}_{\text {mean }}=4.38$. As regards the MAPE coefficient, $\mathrm{MAPE}_{\text {mean }}=0.59$ for LM, MAPE ${ }_{\text {mean }}=1.14$ for $\mathrm{SCG}$, and $\mathrm{MAPE}_{\text {mean }}=1.56$ for CGP. Concerning the $\mathrm{COV}$ coefficient, $\mathrm{COV}_{\text {mean }}=0.14$ for $\mathrm{LM}, \mathrm{COV}_{\text {mean }}=0.18$ for SCG, and $\mathrm{COV}_{\text {mean }}=$ 0.35 for CGP. So, the best values mentioned are for the LM algorithm, they are closer to zero compared to the values obtained by the other two algorithms. So, this algorithm is the best in terms of speed, efficiency and certainty.

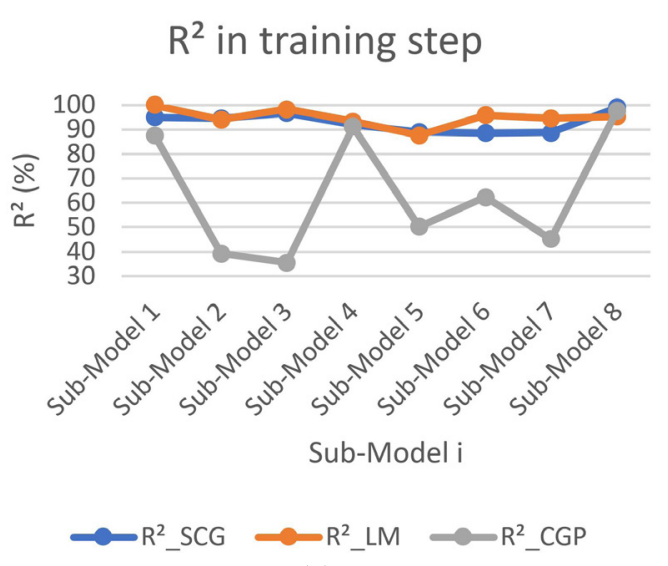

(a)

MAPE in training step

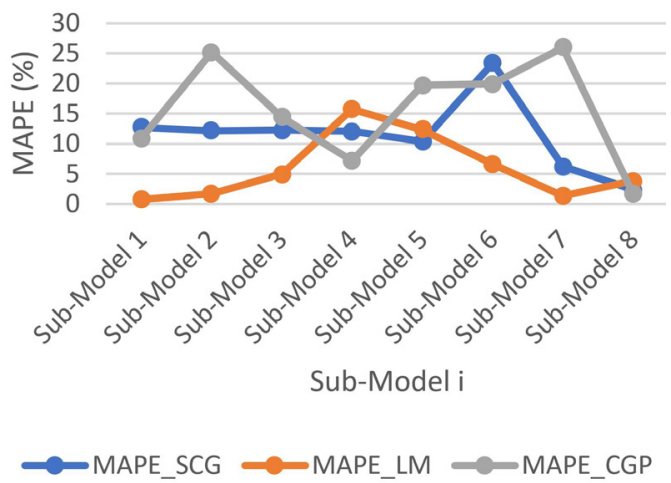

(b)

COV in training step

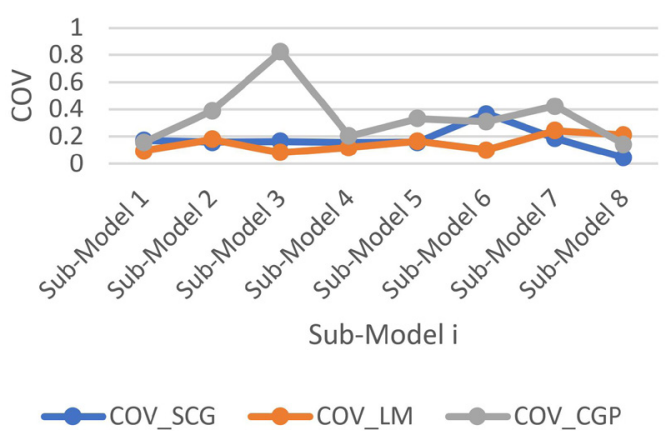

(c)

RMSE in training step

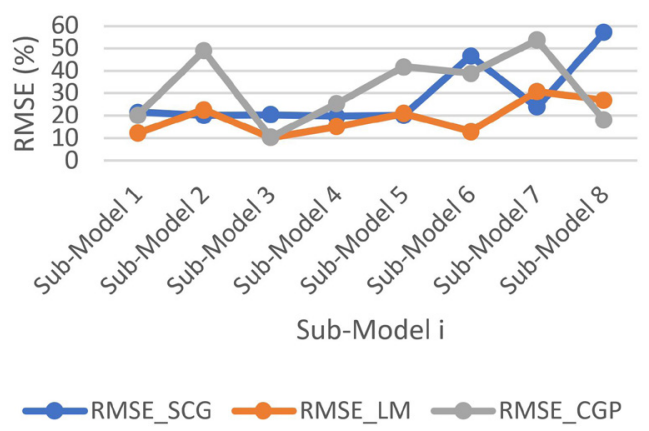

(d)

Figure 13. Prediction in training step of three Algorithms for: (a) Absolute Fraction of Variance $\left(\mathrm{R}^{2}\right)$, (b) Mean Absolute Percentage Error (MAPE), (c) Coefficient of Variation (COV) and (d) Root Mean Squared Error (RMSE) 


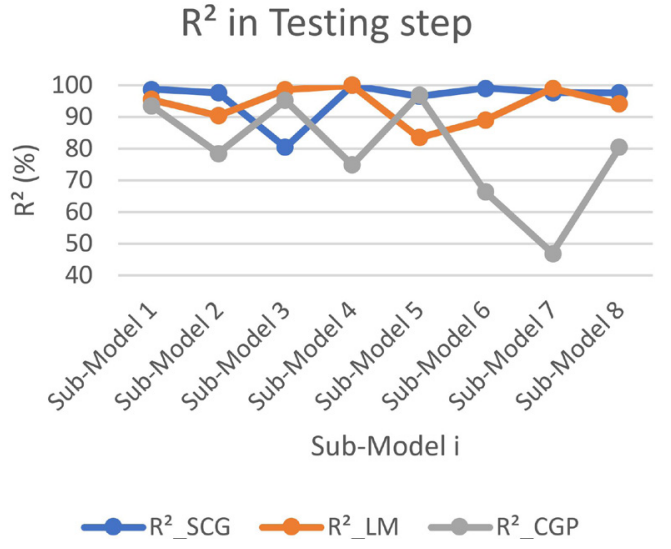

(a)

MAPE in Testing step

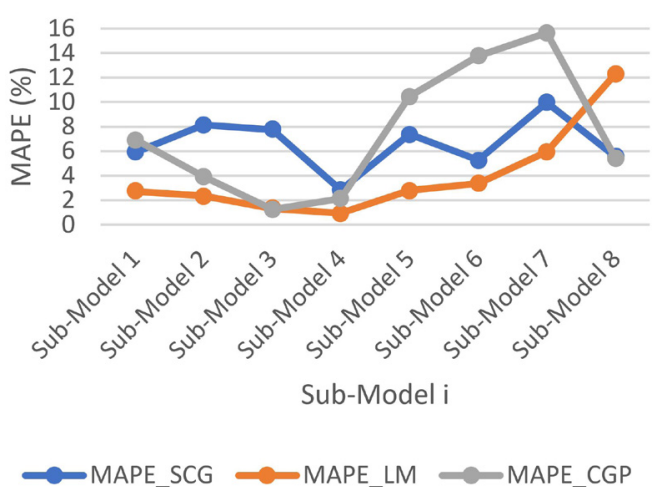

(b)

COV in Testing step

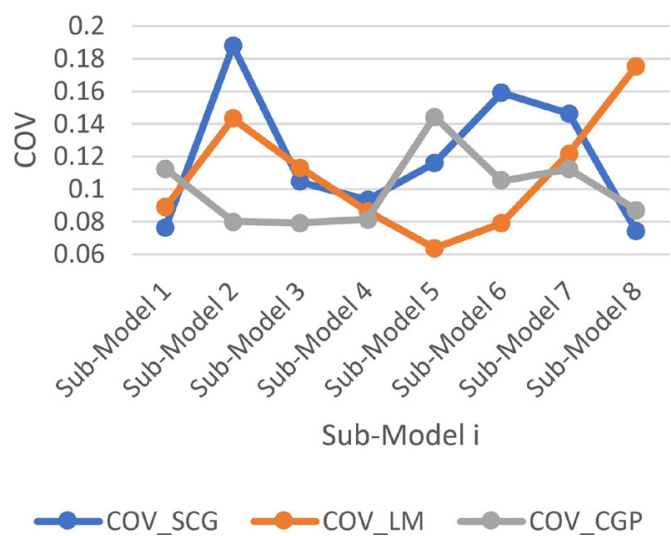

(c)

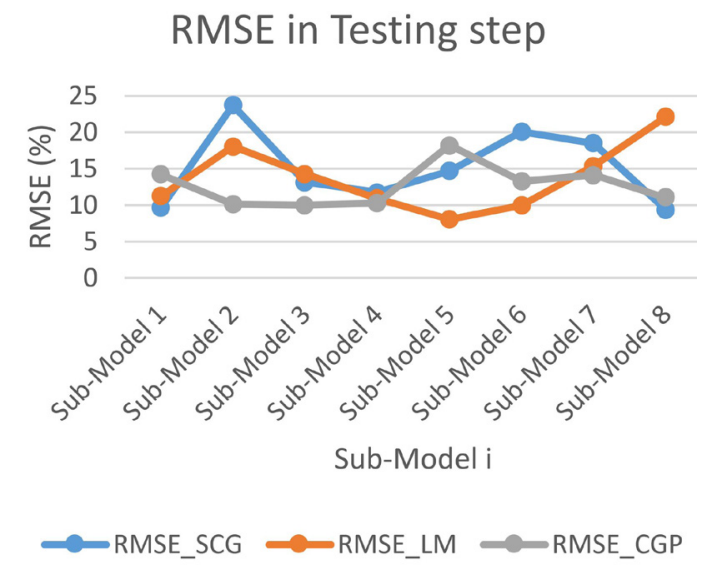

(d)

Figure 14. Prediction in testing step of three Algorithms for: (a) Absolute Fraction of Variance $\left(\mathrm{R}^{2}\right)$, (b) Mean Absolute Percentage Error (MAPE),

(c) Coefficient of Variation (COV) and (d) Root Mean Squared Error (RMSE)

After having processed the best algorithm for applying the proposed ANN method to the SOLON $55 \mathrm{~W}$ photovoltaic panel, one should specify the best choices for one hidden layer and two hidden layers for the best LM algorithm. Table 3 illustrates the values of the different characteristics of the LM algorithm of the coefficient $\mathrm{R}^{2}$ for two different numbers of hidden layers simulated under MATLAB / Simulink based on the data acquired from the SOLON 55W panel. These simulation results demonstrate that the coefficients (training, validation, test and all) have higher values for a single hidden layer than for two hidden layers. The following figures represent the average value of the different characteristics of the coefficient $\mathrm{R}^{2}$ for the single hidden layer case (training ${ }_{\text {mean }}$ $=0.964$, validation mean $_{\text {man }}=0.963$, test mean $_{\text {man }}=0.961$, and all ${ }_{\text {mean }}=0.952$ ) and for two hidden layers (training $_{\text {mean }}=0.883$, validation mean $=0.985$, test mean $_{\text {man }}$ $=0.864$, and all $1_{\text {mean }}=0.886$ ). Therefore, the use of a single hidden layer gives better results, based

Table 3. ANN Training, Validation and Testing Stage Error Values for the LM algorithm

\begin{tabular}{|c|c|c|c|c|c|c|}
\hline \multirow{2}{*}{$\begin{array}{c}\text { Sub- } \\
\text { Models }\end{array}$} & Hidden & \multirow{2}{*}{$\begin{array}{c}\text { Nomber of Neurons } \\
\text { layers }\end{array}$} & \multicolumn{4}{|c|}{$\mathbf{R}^{2}$} \\
\cline { 4 - 7 } 1st hidden, 2nd hidden $\}$ & Training & Validation & Testing & All \\
\hline 1 & 1 & 4 & 99.866 & 99.935 & 95.491 & 96.046 \\
\hline 2 & 1 & 6 & 93.973 & 88.604 & 90.389 & 92.612 \\
\hline 3 & 1 & 7 & 98.346 & 97.866 & 98.601 & 97.02 \\
\hline 4 & 1 & 9 & 93.325 & 98.661 & 99.948 & 95.045 \\
\hline 5 & 2 & $\{2,1\}$ & 87.555 & 97.197 & 83.511 & 89.198 \\
\hline 6 & 2 & $\{2,3\}$ & 95.753 & 97.306 & 89.06 & 95.169 \\
\hline 7 & 2 & $\{3,2\}$ & 74.539 & 99.738 & 79.018 & 78.111 \\
\hline 8 & 2 & $\{5,4\}$ & 95.336 & 99.976 & 94.056 & 92.032 \\
\hline
\end{tabular}


on the coefficients already mentioned, for the training, validation and testing stages.

Figure 15 illustrates the simulation of the different evaluations of the proposed model based on Table 3 .

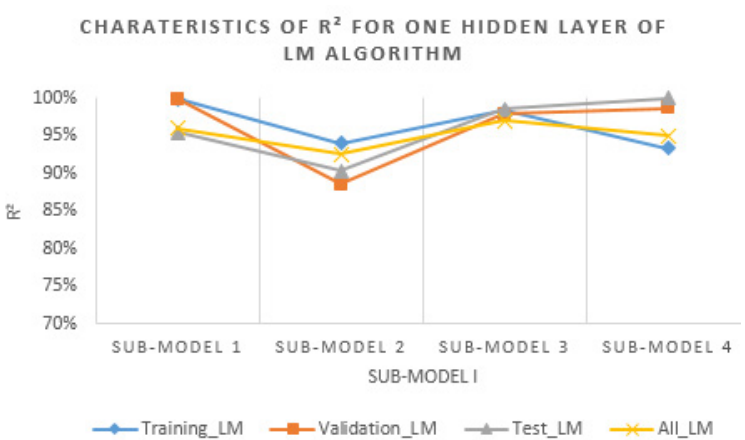

(a)

Charateristics of $\mathrm{R}^{2}$ for two Hidden Layers of LM algorithm

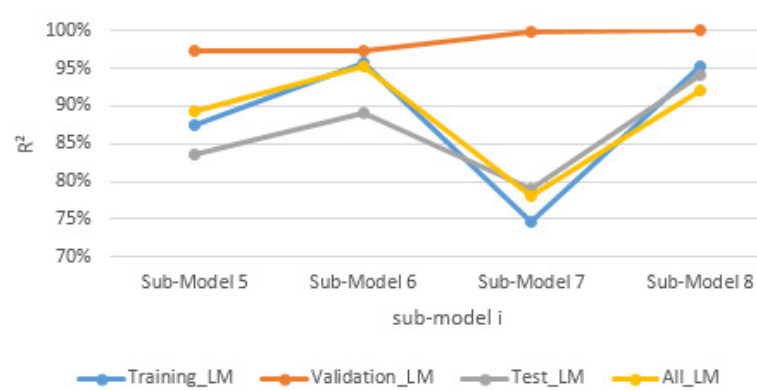

(b)

Figure 15. Characteristics of Absolute Fraction of Variance $\left(\mathrm{R}^{2}\right)$ of $\mathrm{LM}$ algorithm, (a) for one Hidden Layer. (b) two Hidden Layers

The simulation results in Table 3 summarize a difference in processing between the number of layers. For sub-models 1, 2, 3 and 4, which represent a single hidden, the results of "Training" and "Testing" are equal to $99 \%$. For sub-models 5, 6, 7 and 8 which represent two layers hidden, the "Training" and "Testing" results are equal to $95 \%$. Based on the results found under MATLAB / Simulink, it can be stated that the new "Multi-Input Multi-Layer One-Output" (MIMLOO) approach applied to the SOLON 55W photovoltaic panel after data acquisition gives a better estimate, which is obtained by calculating the different performances of the ANN method. This approach summarizes the choice of the number of hidden layers, the different number of neurons and the type of the adequate algorithm.

As it was discussed, the new hybrid machine learning algorithm developed in this section is an overlay of 8 neural sub-models with 2 inputs: Irradiation and temperature. Each of these submodels has a different operating sub-space which is due to the variation in amplitude of the recorded meteorological variables, which change from month to month. The estimates of the 8 neural submodels, when compared to the $\mathrm{P}_{\max }$ test values, show a good fit where the statistical indicators, COV, RMSE, MAPE and $\mathrm{R}^{2}$, vary from one sub-model to another as they are affected by the monthly variation. Figures 16, 17 and 18 illustrate the performance of the proposed machine learning algorithm for the months 5, 6 and 7 of 2019. The results demonstrate the best performance of the proposed approach in estimating the optimal power of the PV generator. On the other hand, the couples of the measured and estimated data for the global model show a high correlation coefficient, which is approximately $97.16 \%$ on average.

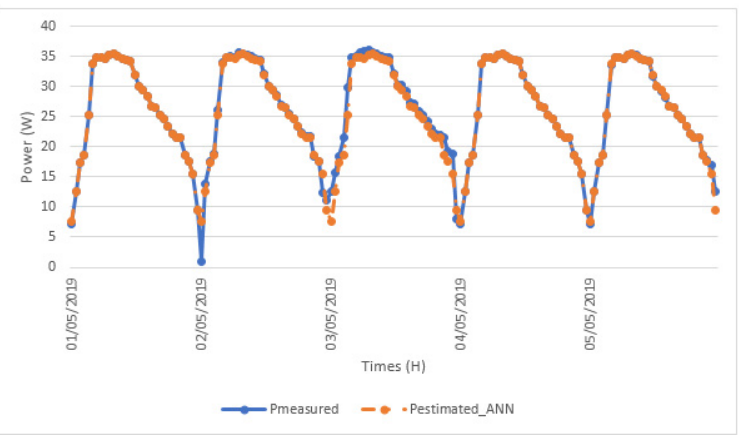

Figure 16. The performances of the learning algorithm for the month May of 2019

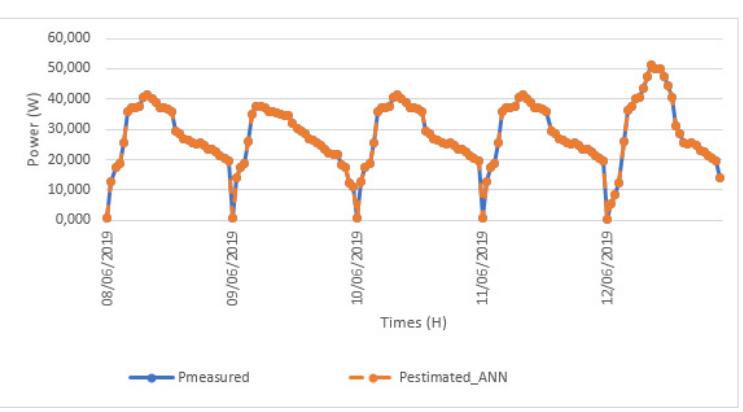

Figure 17. The performances of the learning algorithm for the month June of 2019

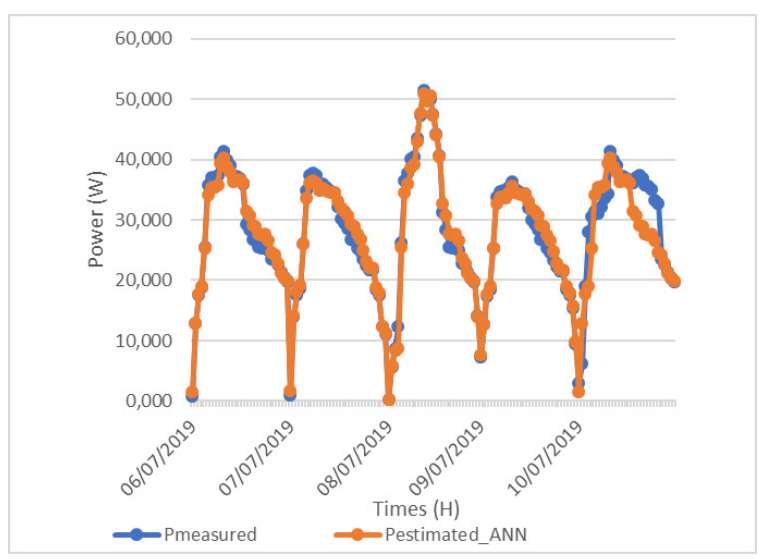

Figure 18. The performances of the learning algorithm for the month July of 2019 


\section{Conclusion}

The present study focuses on the estimation of the optimal power of "SOLON 55W" PV generator, using a new hybrid machine learning algorithm called multi-neural approach. The proposed model is based on the decomposition of the operating space into several sub-spaces allowing the description of the system by several sub-models of simple structure. The output of the overall model is obtained by combining the outputs of all the sub-models. Three backpropagation algorithms, namely, Levenberg-Marquardt, scaled conjugate gradient and Pola-Ribiere Conjugate Gradient algorithm, are tested in this analysis, which demonstrates the robustness of the Levenberg-Marquardt algorithm for training all sub-models. For training and testing of the proposed model, the data is recorded from sunset to sunrise for 4 months. In addition, a relevance analysis of the input variables generates several multi-neuron configurations and highlights the most critical parameters. For the "SOLON $55 \mathrm{~W}$ " PV generator, the use of the two parameters (Irradiation and temperature) represents the best input scenario to further increase the performance of the multi-neuronal model. Using this new approach speeds up the training phase and ensures the model's precision stability throughout the year. In addition, it is a good tool for monitoring the quality of the estimate and detecting if there are any decreases. Subsequently, it is easy to locate the sub-model where there is a deficiency and try to adjust its parameters without affecting the other parameters of the sub-models, which is impossible when structural models based on a unique structure are used. Generally, with regard to statistical indicators known as performance metrics allowing the comparison between different approaches, the results obtained demonstrate the efficiency of the proposed approach compared to different machine learning algorithms developed as part of certain contributions to the literature for different climatic variations.

\section{REFERENCES}

Alsina, E. F., Bortolini, M., Gamberi, M. \& Regattieri, A. (2016). Artificial neural network optimisation for monthly average daily global solar radiation prediction, Energy Conversion and Management, 120, 320-329.

Boukli, H. (2011). Conception et Réalisation d'un Générateur photovoltaïque Muni d'un convertisseur MPPT pour une Meilleure Gestion Energétique. These de magister, Université Abou-Baker Blkaid-Tlemcen.

Gani, A., Mohammadi, K., Shamshirband, S., Khorasanizadeh, H., Danesh, A. S., Piri, J., Zuraini, I. \& Zamani, M. (2016). Day of the year-based prediction of horizontal global solar radiation by a neural network auto-regressive model, Theoretical and Applied Climatology, 125, 679-689. DOI: 10.1007/s00704-015-1533-8

Hajdarevic, A., Banjanovic-Mehmedovic, L., Dzananovic, I., Mehmedovic, F. \& Ayaz Ahmad, M. (2015). Recurent Neural Network as a Tool for Parameter Anomaly Detection in Thermal Power Plant, International Journal of Scientific \& Engineering Research, 6(8), 448-455.

Han, Y., Zhang, D., Zhang, H. \& Gao, Q. (2016). Survey of Maximum Power Point Tracking Techniques for Photo-Voltaic Array, International Journal of Control and Automation 9(8), 49-58. DOI: 10.14257/ ijca.2016.9.8.06
Hananou, F. \& Rouabah, A. (2014). Modélisation et simulation d'un système photovoltäqque. Master académique, Université Kasdi Merbah Ouargla.

Hyndman, R. J. \& Koehler, A. B. (2006). Another look at measures of forecast accuracy, International Journal of Forecasting, 22(4), 679-688. DOI: 10.1016/j.ijforecast.2006.03.001

Jalal, M. A., Chabaa, S. \& Zeroual, A. (2019). A new artificial multi-neural approach to estimate the hourly global solar radiation in a semi-arid climate site, Theorical and Applied Climatology, 139, 1261-1276. DOI: 10.1007/s00704-019-03033-1

Kalogirou, S. A. (2004). Optimization of solar systems using artificial neural-networks and genetic algorithms, Applied Energy, 77(4), 383-405.

Kok, B. C., Goh, H. H. \& Chua, H. G. (2012). Optimal Power Tracker for Stand-Alone Photovoltaic system using Artificial Neural Network (ANN) and Particle Swarm Optimization (PSO). In International Conference on Renewable Energies and Power Quality (ICREPQ'12) Santiago de Compostela (Spain), 1(10), (pp. 440-445).

Kumar, N., Sharma, S. P., Sinha, U. K. \& Nayak, Y. (2016). Prediction of Solar Energy Based on Intelligent ANN Modeling, International Journal of Renewable Energy Research - IJRER, 6(1), 183-188. 
Li, F., Wang, S. \& Wei, J. (2018). Long term rolling prediction model for solar radiation combining empirical mode decomposition (EMD) and artificial neural network (ANN) techniques, Journal of Renewable and Sustainable Energy, 10(1), 013704. DOI: $10.1063 / 1.4999240$

Luque, A. \& Hegedus, S. (2003). Handbook of photovoltaic Science and Engineering. John Wiley \& Sons Ltd.

Madani, K. \& Thiaw L. (2007). Self-organizing multi-modeling: A different way to design intelligent predictors, Neurocomputing, 70(16-18), 2836-2852. DOI: 10.1016/j.neucom.2006.06.016

Manan, M., Birinchi, B., Sahaj, S. \& Anshu, M. (2018). Performance prediction of PV module using electrical equivalent model and artificial neural network, Solar Energy, 176, 104-117.

McCulloch, W. S. \& Pitts, W. (1943). A logical calculus of the ideas immanent in nervous activity, The Bulletin of Mathematical Biophysics, 5, 115-133.

Mellit, A., Shaari, S., Mekki, H. \& Khorissi, N., (2008). FPGA based Aritificial Neural Network for Prediction of Solar Radiation Data from Sunshine Duration and Air Temperature. In IEEE Region 8 International Conference on Computational Technologies in Electrical and Electronics Engineering (pp. 118-123).

Murat, Y. S. (2006). Comparison of fuzzy logic and artificial neural networks approaches in vehicle delay modeling, Transportation Research Part C: Emerging Technologies, 14(5), 316-334.

Ndoye, S., Ibrahima, L. Y., Fabé Idrissa, B., Ould Habiboulahi, L. \& Grégoire, S. (2009). Modélisation et simulation sous Matlab/Simulink de la chaine d'alimentation d'une station relais de télécommunications en énergie solaire photovoltaïque dans une zone isolée du réseau électrique, Journal des Sciences, 9(2), 45-55.

Ouada, M., Tarfaya, A., Meridjet, M. S. \& Dib, D. (2012). Etude et Caractérisation sous Matlab/Simulink d'un Générateur Photovoltaïque, The 2nd International Seminar on New and Renewable Energies.

Pontius, R. G., Thontteh, O. \& Chen, H. (2008). Components of information for multiple resolution comparison between maps that share a real variable, Environmental and Ecological Statistics, 15(2), 111142. DOI: $10.1007 / \mathrm{s} 10651-007-0043-\mathrm{y}$
Pushpi, R. \& Dilip, K. S. (2018). Artificial Neural Networks approach induced by fuzzy logic for traffic delay estimation, Journal of Engineering Technology, 6(2), 127-141.

Rumelhart, D. E., Hinton, G. E. \& Williams, R. J. (1986). Learning representations by backpropagating errors, Nature, 323, 533-536.

Soria, Z. \& Makhlouf, C. (2017). Etude et Simulation d'un Générateur Photovoltaïque Muni d'un Convertisseur MPPT pour une Meilleur Gestion Energétique. Faculté de Technologie, Département d'Électronique. Universite Mohamed Boudiaf - M'sila.

Soteris, K. (2009). Solar Energy Engineering. Processes and Systems, 1st ed. Academic Press.

Sözen, A., Erol, A. \& Mehmet, Ö. (2004). Estimation of solar potential in Turkey by artificial neural network using meteorological and geographical data, Elsevier Science: Energy Conversion and Management, 45, 18-19.

Takun, P., Kaitwanidvilai, S. \& Jettanasen, C. (2011). Maximum power point tracking using fuzzy logic control for photovoltaic systems. In Proceedings of the International Multi Conference of Engineers and Computer Scientists IMECS, Vol II (pp. 986-990).

Taright, Y. \& Hubin, M. (1998). FPGA implementation of a multilayer perceptron neural networkusing VHDL. In ICSP'98, Fourth International Conference on Signal Processing (Cat. No.98TH8344), (1311-1314).

Timilsina, G. R., Kurdgelashvili, L. \& Narbel, P. A. (2012). Solar energy: markets, economics and policies, Renewable and Sustainable Energy Reviews, 16(1), 449-465. DOI: 10.1016/j.rser.2011.08.009

Willmott, C. J. \& Matsuura, K. (2006). On the use of dimensioned measures of error to evaluate the performance of spatial interpolators, International Journal of Geographical Information Science, 20, 89102. DOI: $10.1080 / 13658810500286976$

Wu, Y., Wang, H., Zhang, B. \& Du, K. L. (2012). Using radial basis function networks for function approximation and classification, ISRN Applied Mathematics, Volume 2012, Article ID 324194, 34 pp. DOI: $10.5402 / 2012 / 324194$ 